\title{
CORRELACIÓN ENTRE RASGOS DE MASCULINIDAD-FEMINIDADY SATISFACCIÓN MARITAL EN MIGRANTES MEXICANOS
}

\author{
ANGÉLICA OJEDA GARCÍA \\ LIZBETH MELBY CALCÁNEO \\ VANESA SÁNCHEZ SOTO \\ MARIANA RODARTE DE LARA
}

RESUMEN. El propósito de esta investigación es evaluar los rasgos de masculinidad-feminidad predominantes entre los miembros de la pareja migrante, a fin de conocer aquéllos que favorecen su satisfacción marital. Para ello, se utilizó un muestreo no probabilístico de tipo intencional conformado por 150 migrantes. Los resultados muestran que éstos suelen tomar lo positivo de ambos rasgos de género y se adjudican características de tipo masculino como predominantes, auto-describiéndose como andróginos positivos. No obstante, mientras en los hombres existe una tendencia a lo positivo, en las mujeres se da hacia lo negativo. Con respecto a la satisfacción marital, los esposos resultaron satisfechos, tanto en la parte instrumental como en la emocional; mientras que las esposas reportaron estar significativamente satisfechas únicamente con la parte instrumental, por lo que reflejan insatisfacción marital. Esto indica que el rasgo de masculinidad positiva en ellas ejerce una correlación negativa respecto a la forma en que perciben su relación de pareja. PALABRAS ClAVE. Masculinidad, feminidad, satisfacción marital, migración, pareja.

ABSTRACT. This article examines the predominant attributes of masculinity and femininity at immigrant married couples in order to know which attributes favourate their marital satisfaction. For this purpose we used a non probabilistc model. The results showed that this individuals tend to take what is positive from both gender attributes and they give to themselves masculine attributes as predominant and as positive androgynies. Nevertheless, men tend to incline to positiveness while women to negativiness attributes. With respect to the marital satisfaction, husbands turned out to be satisfied by both areas: in the instrumental part and the emotional part; while wives were reported to be satisfied only in the instrumental one. Thus, it can be said that women feel that their couple relationship is unsatisfactory. Which in turn shows that the positive masculine attributes in women produced a negative correlation, with regard of the way they perceive a couple relationship.

KEY WORDS. Masculinity, femininity, marital satisfaction, immigration, couple. 


\section{INTRODUCCIÓN}

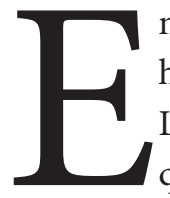

n un principio, se mantuvo la creencia que las diferencias encontradas entre hombres y mujeres son «naturales» y genéticamente determinadas (DíazLoving, Aragón \& Sánchez, 1994: 180). Tal visión tradicionalista plantea, lo que es ser hombre y lo que es ser mujer, como dos personalidades opuestas de un mismo continuo y en cuya interacción se derivan relaciones adecuadas o apropiadas entre los mismos (Gilligan, 1982:173). A partir de esta postura, en la literatura se manejan distintas terminologías para hacer referencia a dichos extremos: instrumentalidadexpresividad, dominación-subordinación, masculino vs. femenino. De tal suerte que se plantea la existencia de dos mundos distintos y separados: el de lo masculino, al cual se le conoce como «Masculinidad» y el de lo femenino, conocido como «Feminidad» (Maldonado, García y García, 1994: 753).

El análisis de estos rasgos, llamados de masculinidad-feminidad, de la diferenciación entre las características que son consideradas, socialmente, apropiadas para ambos sexos y de las expectativas adjudicadas a dichos mundos, establecen e integran distintos roles de funcionamiento específicos, que determinan la dinámica interna de la vida conyugal, familiar y social (Bustos, 1994: 755).

Rocha (2004: 1) hace toda una revisión literaria acerca de cómo debe comportarse y expresarse cada «mundo», tanto a nivel individual (de identidad), como social (de estereotipo). La autora encuentra que, en un primer acercamiento, quienes poseen rasgos femeninos se les ha adjudicado el desempeño de un papel: dependiente, decorativo, cálido, expresivo, interesados en los demás, apoyadores y casi nunca en una posición de autoridad. Mientras que, a quienes poseen rasgos masculinos, se les describe como: independientes, activos, en puestos de mando, asertivos, racionales y orientados hacia la actividad instrumental. Planteamiento que hoy se sigue retomando para hacer análisis y estudios diferenciales por género.

En una segunda visión, el Modelo Bipolar (Brehm, 1992: 161) reafirma la existencia de dos mundos que nunca se tocan, en donde la bipolaridad está dada por la imposibilidad teórica de que en un solo individuo aparezcan, en forma amplia y desarrollada, características tanto masculinas como femeninas al mismo tiempo. En consecuencia, a mayor masculinidad menor femineidad $y$, a mayor femineidad, menor masculinidad. En la actualidad, el uso de este modelo ha sido restringido, como consecuencia de las transformaciones sociales, el desempeño de funciones, de las tareas desempeñadas y el contexto en el que se den, dando lugar a un nuevo término, al que se le denomina «Androginia» (Brehm, 1992: 404; Hoffman, 2000: 476).

Bajo la visión de la existencia de ambos rasgos de género en una persona, Rocha (2004: 84) sostiene que lejos de ser polos opuestos, los rasgos de feminidad-masculinidad comparten características similares, es decir, un mismo descriptor puede se visto socialmente aceptable o no para un hombre, que para una mujer. Al respecto, Brehm (1992: 136) y Hoffman (2000: 476) refieren que, bajo este enfoque dualista, se acepta el supuesto de que existan tanto hombres como mujeres altos y bajos en características 
masculinas y femeninas, en cuya interacción o combinación darían por resultado cuatro formas de evaluar o percibir la dinámica conyugal y, por ende, su nivel de percepción de satisfacción marital: Masculinos Positivos, Masculinos Negativos, Femeninos Positivos y Femeninos Negativos, cuya combinación puede dar resultado a otras dos combinaciones: Andróginos e Indiferenciados, los cuáles se refieren a que la persona presente ambos rasgos de masculinidad-femineidad altos o bajos, respectivamente.

Díaz-Loving, Rivera y Sánchez (2001: 134-137), hicieron un estudio para explorar las características socialmente deseables e indeseables en hombres y mujeres mexicanas, en donde obtuvieron cuatro factores, mismos que han sido complementados y reforzados por nuevas características (ver tabla 1).

TABLA 1

Distribución por adjetivos descritos en la literatura como pertenecientes a uno u otro factor de masculinidad-femineidad

\begin{tabular}{|c|c|c|c|c|}
\hline \multicolumn{2}{|c|}{$\begin{array}{l}\text { MASCULINIDAD } \\
\text { POSITIVA }\end{array}$} & $\begin{array}{l}\text { FEMINEIDAD } \\
\text { POSITIVA }\end{array}$ & $\begin{array}{l}\text { MASCULINIDAD } \\
\text { NEGATIVA }\end{array}$ & $\begin{array}{l}\text { FEMINEIDAD } \\
\text { NEGATIVA }\end{array}$ \\
\hline Capaz & Autónomo & Amable & Grosero & Chillona \\
\hline Decidido & Asertivo & Afectuosa & Autoritario & Crédula \\
\hline Independiente & Sociable & Tierna & Malvado & Débil \\
\hline Competitivo & Valiente & Sincera & Violento & Aprovechada \\
\hline Reflexivo & Vanidoso & Tranquila & Conformista & Sufrida \\
\hline Exitoso & Persistente & Atenta & Altanero & Intolerante \\
\hline Preparado & Determinado & Generosa & Dominante & Cobarde \\
\hline Cumplido & Organizado & Gentil & Conflictivo & Audaz \\
\hline Inteligente & Leal & Cooperadora & Envidioso & Servil \\
\hline Educado & Honrado & Emocional & Arrogante & Indecisa \\
\hline Astuto & Cumplido & Educada & Abusivo & Miedosa \\
\hline Luchón & Honesto & Cálida & Chismoso & Voluble \\
\hline Proveedor & Autosuficiente & Dulce & Inestable & Insegura \\
\hline Emprendedor & Trabajador & Comprensiva & Egoísta & Manipuladora \\
\hline Hábil & Firme & Cariñosa & Dictatorial & \\
\hline \multirow[t]{9}{*}{ Ordenado } & & Paciente & Vengativo & \\
\hline & & Compartida & Corrupto & \\
\hline & & Amorosa & Vengativo & \\
\hline & & & Flojo & \\
\hline & & & Tenaz & \\
\hline & & & Ambicioso & \\
\hline & & & Chismoso & \\
\hline & & & Mentiroso & \\
\hline & & & Quejumbroso & \\
\hline
\end{tabular}

Nota: los adjetivos calificativos se anotan indistintamente el género, en masculino o femenino. Fuentes: Carrillo, Cortés, Flores y Reyes, 2000; Díaz-Loving y Rocha, 2004; Polanco, 2004. 
Donde masculinidad positiva se refiere a aquellos rasgos que favorecen el desempeño laboral, el trabajo cognoscitivo, el logro y la competitividad; además de ser caracterizados por adjetivos que se consideran habilidades o «instrumentos» para alcanzar dicha meta previa.

Femineidad positiva son aquellas características que dirigen la energía hacia buscar el bienestar de los otros, que favorecen las relaciones comunales e interpersonales, que incitan la armonía del grupo, por lo que se consideran de tipo afectivo-comunales y que son las socialmente deseables.

Masculinidad negativa, incluye rasgos de tipo instrumental que desfavorecen la productividad, la autosuficiencia y autonomía de la persona.

Femineidad negativa, se refiere a todas aquellas características de tipo afectivas que desfavorecen las relaciones interpersonales y la salud social.

Las correlaciones hechas por autores como Brehm (1992: 137) y Hoffman (2000: 496), para ambos sexos, muestran que poseer características femeninas o masculinas negativas es lo más desfavorable para que la pareja logre una satisfacción marital, mientras que poseer características femeninas y/o masculinas positivas la favorece. Algunos otros autores (p.e., Diener \& Suh, 1999: 484; Veenhoven, 2005: 331) opinan que la estabilidad marital también se ve influida por factores externos de los lugares donde residen.

De acuerdo con Díaz-Loving, Ruiz, Cárdenas, Alvarado y Reyes (1994: 138-139) la misma evolución cultural ha hecho que las mujeres desarrollen sus habilidades instrumentales, mientras que los hombres fortalecen sus características femeninas positivas y engrandecen su capacidad de expresión emocional. Esta situación, dentro del contexto de la migración, se confirma pues, por un lado, las mujeres que se quedan en el lugar de origen se ven en la necesidad de sobrellevar su doble rol parental, como resultado de que sus maridos están ausentes y ellas tienen que cumplir con las funciones de madre y padre a la vez; por otro, como consecuencia del estado de soledad al que el proceso de migración los conduce, los hombres han tenido que aprender a expresar sus emociones y trabajar arduamente, para generar un cambio en su estilo de vida e ir en busca de mejores oportunidades (Sánchez de la Barquera, 1997: 5). Sin embargo, sólo unos pocos logran conseguir trabajo y otros más mantenerlo, así como cumplir con lo que se espera de ellos, o bien, con los requerimientos que, la cultura que los acoge, les solicita (Finch, Catalano, Novaco y Vega, 2003: 188).

Díaz-Guerrero (1994) hizo un estudio comparativo del concepto «respeto», tanto en individuos anglosajones como mexicanos, en donde encontró que mientras para los estadounidenses, una relación de respeto es descrita como una relación entre iguales e impersonal, para los mexicanos, el término es asociado con la idea de obediencia, protección y/o una relación extremadamente íntima, al grado de involucrar sentimientos personales. A partir de esos datos, hizo una diferenciación entre las personalidades de los mexicanos y norteamericanos, en donde encontró que estos últimos tienden a ser más activos en su estilo de confrontación a desafíos y problemas de la vida, prefieren modificar al ambiente, a la sociedad o a otros. La agresividad, el enojo, el desprecio, la determinación, la envidia, el odio, el dolor, el orgullo, la actitud individualista, la competitividad, la actitud positiva, la seguridad en sí mismos, se conciben como caracterís- 
ticas típicas de ellos. Mientras, los mexicanos tienden a modificarse a sí mismos y a ser más pasivos ante el manejo de los problemas cotidianos. La abnegación en la madre, la obediencia de los hijos, el propio sacrificio en todos, la sumisión, la dependencia, las buenas maneras, la cortesía, el aguante, la «concha», la vergüenza, la devoción, la simpatía, centrados en la familia, el pesimismo, la cooperación y el fatalismo, pueden ser considerados como virtudes socioculturales mexicanas.

En Estados Unidos tenderán a producir individuos que son activos, independientes, individualistas, autónomos, orientados hacia el logro, competitivos, firmes, hasta cierto punto impulsivos, tensos y agresivos, donde resalte la fuerza, la acción, el poder, el producir y el lograr. Mientras que el patrón mexicano producirá individuos complacientes, obedientes, afiliativos, interdependientes, flexibles, cooperativos, no orientados hacia el logro, más bien reposados y que socialmente aprueben ser obedientes, predispuestos al servilismo y a la corrupción; sus atributos más sobresalientes abarcan la razón, lo racional, el pensar y la inteligencia.

Hay literatura que apunta que el hecho de que en los integrantes de una relación de pareja predominen rasgos masculinos o femeninos negativos, les genera cierto estrés psicológico (Frone, Markel \& Yardley, 1997: 145), baja autoestima (Courmoyer \& Mahalik, 1995: 17), ansiedad y depresión (Sharpe, Heppner, Dixon 1995: 3), síntomas obsesivos-compulsivos (Hayes \& Mahalik, 2000: 118) e incluso dificultad para establecer y mantener relaciones cercanas, altos niveles de intimidad (Fischer \& Good, 1997: 167) y ajuste marital (Campbell \& Snow, 1992: 86-87), entre otros. En cuanto a quienes viven el proceso de la migración, Vinokur, Price y Caplan (citado en Finch, et. al., 2003: 181,182) encontraron que la pérdida del empleo conducía, a los afectados, a estar enojados, irritables, críticos y a insultar a sus esposas, esposos o parejas.

Otro estudio al respecto es el de Kanaiaupuni y Shwan (2000: 1314) que se interesan por hacer una diferenciación entre las relaciones que establecen hombres y mujeres, partiendo de lo que implica y significa ser hombre o mujer en el contexto de la migración, pues, para ellos, el comportamiento migrante está determinado, en gran medida, por el sexo biológico de los individuos que, a su vez, viene arraigado y establecido por los sistemas familiares y la sociedad. Parrado y Flippen (2005: 627) sostienen que el proceso de la migración, invariablemente, es perjudicial para el vínculo social e interconexión entre hombres y mujeres, particularmente para las mujeres, pues a éstas las hace más dependientes de sus esposos y, ellas, además deberán trabajar en la reconstrucción de esos vínculos sociales, dentro y fuera de casa.

Es necesario considerar que el contexto social cambia la percepción, la interpretación, el significado, así como el ejercicio de los rasgos que distinguen a ambos sexos, lo que, a su vez, marca pautas de interacción social entre hombres y mujeres. De ahí que la pregunta que sería interesante plantear es «¿cuáles son las características típicas o ideales que manifiestan un hombre y una mujer dentro de una relación de pareja, como indicadores que permiten o no el crecimiento de ésta, con la finalidad de responder si sería más sencillo o complicado interactuar entre personas con rasgos de masculinidad o femineidad similares o complementarios?». Esta pregunta es la guía de este trabajo enfocado, de manera muy somera, a una población específica: individuos migrantes con pareja. 
La idea de crecimiento, en este trabajo, se analizará bajo el constructo de Satisfacción Marital, vista ésta como el producto en el que se conjugan ambos rasgos, masculinos y femeninos. De tal forma que el primer objetivo de la presente investigación fue responder la siguiente cuestión, «¿cuáles son los rasgos de masculinidad-femineidad que predominan entre los miembros de la pareja migrante?» A partir de esta pregunta se respondería una segunda, «¿los hombres siguen siendo culturalmente masculinos y las mujeres femeninas?» Finalmente, buscamos responder «¿cuál de las combinaciones entre rasgos masculinos-femeninos predominantes favorece la satisfacción marital, en una muestra de migrantes mexicanas hacia los EU?».

\section{Método}

La muestra estuvo constituida por 150 sujetos ( 88 hombres y 62 mujeres), todos con relación de pareja permanente, con el entendido de que, por lo menos, contaban un año de vivir juntos. De ellos, 100 casados legalmente y 50 en unión libre, todos bajo la situación de migración. El rango de años de casados iba de 1 hasta los 33 años, con un promedio de 9.44 años y una desviación estándar de 7.4. El 65\% (97) fue de Miami, Florida y el 30\% (53) de Atlanta, Georgia. El rango de edad iba de 15 hasta 70 años, con una media de 32 años y una desviación estándar de 10. La escolaridad partía de primaria incompleta hasta universidad completa, con una media entre secundaria completa y preparatoria incompleta. Las reglas de inclusión fueron que los participantes se encontraran, actualmente, viviendo bajo en mismo techo con una pareja y que respondieran a todo el instrumento que se les aplicó. El muestreo fue no probabilístico de tipo intencional.

\section{Instrumentos}

Se aplicó la Escala de Roles de Género de Polanco (2004: 122-132). De los 47 rasgos que inicialmente lo conformaron, por análisis de discriminación se redujo a 39 características en formato Likert pictórico, con 5 opciones de respuesta que van de Nada (1) a Muchísimo (5), con una Confiabilidad total de $\alpha=.7605$, compuesto de 5 dimensiones que explican el 53.2\% de la varianza: Instrumental Positivo $(\alpha=.8808)$, Expresivo Social Afectivo ( $\alpha=.8982)$, Expresivo Normativo $(\alpha=.7997)$, Atributos Expresivos $(\alpha=.7378)$ y Sociales Tradicionales ( $\alpha=.7355)$, la cual se aplicó completa.

El Inventario Multifacético de Satisfacción Marital (IMSM) de Cortés, Díaz-Loving, Reyes, Rivera y Monjaraz (1994: 125). Del instrumento original en formato Likert, compuesto por 47 afirmaciones distribuidas en 6 factores que explican el $68.8 \%$ de la varianza y con alfas de Cronbach que van de .86 a .92 , se tomaron de 3 a 5 reactivos con cargas factoriales mayores a .40 por factor. Las 20 afirmaciones resultantes se distribuyeron al azar y se diseñaron en un formato con 3 opciones de respuesta: Mucho (3), Poco (2) y Nada (1); se aplicó en versión corta. 


\section{Procedimiento}

Una vez aplicados los instrumentos por cada individuo y para no perder el objeto de este estudio, que fue el evaluar a la pareja migrante, no debemos perder de vista que los entrevistados fueron individuos casados con mínimo de un año de vivir juntos, para luego, con esa misma muestra, conformar parejas por combinación entre roles de género y observar su nivel de satisfacción marital.

\section{Resultados}

En primer lugar, se realizó un análisis de frecuencias para conocer la distribución y discriminación de los reactivos. Posteriormente de los reactivos que si discriminaron, se sometieron a un Análisis Factorial por Componentes Principales para ambas escalas. En el primer caso, se obtuvieron 4 factores que explican el $48.61 \%$ del total de la varian$\mathrm{za}$, con un peso factorial superior a 1 (ver tabla 2).

TABLA 2

Factores resultantes de la Escala de Roles de Género para una muestra de individuos migrantes mexicanos con pareja

\begin{tabular}{|c|c|c|c|}
\hline \multicolumn{2}{|c|}{ MASCULINIDAD } & \multicolumn{2}{|c|}{ FEMINEIDAD } \\
\hline $\begin{array}{c}\text { FACTOR } 1 \text { Masculinidad } \\
\text { Positiva }\end{array}$ & Peso Factorial & $\begin{array}{c}\text { FACTOR 3: } \\
\text { Femineidad Positiva }\end{array}$ & Peso Factorial \\
\hline $\begin{array}{ccc}\mathrm{N}=14 & \mathrm{X}=3.8 & \mathrm{~S}=.07 \\
\mathrm{a}=.885 & \end{array}$ & 1 cso r actoriar & $\begin{array}{lll}\mathrm{N}=11 & \mathrm{X}=4.14 & \mathrm{~S}=.04 \\
& \mathrm{a}=.863 & \end{array}$ & teso tactorlar \\
\hline Autosuficiente & .802 & Amoroso/a & .788 \\
\hline Valiente & .667 & Cariñoso/a & .784 \\
\hline Competente & .650 & Comprensivo/a & .672 \\
\hline Ambicioso/a & .633 & Familiar & .655 \\
\hline Competitivo/a & .616 & Protector/a & .617 \\
\hline Inteligente & .556 & Leal & .547 \\
\hline Proveedor/a & .547 & Fiel & .525 \\
\hline Trabajador/a & .532 & Tolerante & .513 \\
\hline Aguantador/a & .507 & Amable & .469 \\
\hline Educado/a & .489 & Respetuoso/a & .413 \\
\hline Franco/a & .487 & Amigable & .397 \\
\hline Sensible & .449 & & \\
\hline Compartido/a & .430 & & \\
\hline $\begin{array}{l}\text { FACTOR 4: } \\
\text { Masculinidad Negativa } \\
\begin{array}{ccc}\mathrm{N}=7 \quad \mathrm{x}=2.21 \quad \mathrm{~s}=.58 \\
\mathrm{a}=.442\end{array}\end{array}$ & Peso Factorial & $\begin{array}{l}\text { FACTOR 2: } \\
\text { Femineidad Negativa } \\
\mathrm{N}=6 \quad \mathrm{X}=2.33 \quad \mathrm{~s}=.047 \\
\mathrm{a}=.726\end{array}$ & Peso Factorial \\
\hline Flojo/a & .755 & Indeciso/a & .738 \\
\hline
\end{tabular}




\begin{tabular}{|c|c|c|c|}
\hline Egoísta & .563 & Miedoso/a & .697 \\
\hline Aprovechado/a & .530 & Inseguro/a & .629 \\
\hline Sociable (Insociable) & -.513 & Inestable & .555 \\
\hline Chismoso/a & .507 & Cobarde & .533 \\
\hline Luchón/a (Conformista) & -.460 & Agresivo/a & .408 \\
\hline Mandilón/a & .408 & & \\
\hline
\end{tabular}

Nota: Los adjetivos con carga negativa, como es el caso de los adjetivos sociable y luchón(a), se interpretan a la inversa, es decir, con influencia negativa sobre el factor.

Donde el factor de Masculinidad Positiva quedó compuesto por todos aquellos descriptores que hacen referencia a que la persona cuenta con las herramientas, habilidades y capacidades necesarias para lograr ser autosuficiente en la vida: trabajadoras, proveedoras, valientes, inteligentes, que suelen ser competitivas y competentes en el desempeño de sus funciones. Por ello, también se describen como ambiciosas; además, son personas sensibles, educadas, francas, aguantadoras y, sobretodo, compartidas.

El factor de Feminidad Positiva quedó integrado por aquellos adjetivos que, en su conjunto, describen a una persona que da todo por el bien suyo y de su persona, en términos emocionales, de vinculación afectiva y sentimental, por ejemplo: amorosa, cariñosa, comprensiva, protectora, leal, fiel, amable, tolerante, respetuosa, amigable y familiar.

El factor de Feminidad Negativa está constituido por adjetivos que describen a una persona con dificultad para el manejo de sus emociones, así como de situaciones de la vida cotidiana y de pareja; entre tales adjetivos se ubican: indeciso, miedoso, inseguro, inestable, cobarde y agresivo.

El factor de Masculinidad Negativa quedó conformado por reactivos que hablan de una persona que no se interesa por sí misma y sus metas, ni por el bienestar del grupo al que pertenece. Los adjetivos que lo describen son: flojo, mandilón, egoísta, aprovechado, insociables, chismoso y conformista.

Del Análisis de Factorial del Inventario Multifacético de Satisfacción Marital, se obtuvieron dos factores que explican el $78.16 \%$ del total de la varianza y con peso factorial superior a 1 (ver tabla 3$)$.

TABLA 3

Factores resultantes del Inventario Multifacético de Satisfacción Marital para una muestra de individuos migrantes mexicanos con pareja

\begin{tabular}{|c|c|}
\hline FActor $1:$ & Peso Factorial \\
\hline Satisfacción Emocional $\quad \mathrm{N}=15 \quad$ Media=2.17 $\mathrm{S}=.004 \quad \alpha=.980$ & 1.019 \\
\hline La frecuencia con que me besa. & 1.001 \\
\hline La forma en que se interesa en tener relaciones sexuales. & .991 \\
\hline La frecuencia en que me de demuestra el amor que siente por mí. & .911 \\
\hline La forma en que nos divertimos como pareja. & \\
\hline
\end{tabular}




\begin{tabular}{|c|c|}
\hline La sensibilidad con que responde a mis emociones. & .888 \\
\hline La frecuencia con que me abraza. & .859 \\
\hline La forma en que me protege. & .810 \\
\hline La forma en que platica conmigo. & .769 \\
\hline La forma en que me demuestra su comprensión. & .754 \\
\hline Los temas de conversación tan variados que tenemos. & .737 \\
\hline La forma en que se interesa en mis problemas. & .728 \\
\hline La forma en que me demuestra su apoyo. & .662 \\
\hline La manera en que soluciona los problemas conyugales. & .630 \\
\hline La frecuencia en que toma decisiones que nos incumben a los dos. & .618 \\
\hline La forma con que toma decisiones que nos incumben a los dos. & .617 \\
\hline \begin{tabular}{llll}
\multicolumn{5}{l}{ FACTOR 2: } \\
Satisfacción Instrumental & $\mathrm{N}=5 \quad$ Media $=2.09 \quad \mathrm{~S}=.016 \quad \alpha=.914$
\end{tabular} & Peso Factorial \\
\hline La atención que les pone a nuestros hijos. & .939 \\
\hline El trato que les da a nuestros hijos. & .850 \\
\hline La manera en que participa en la educación de nuestros hijos. & .757 \\
\hline La forma en que distribuye el dinero. & .570 \\
\hline La forma en que propone se hagan las tareas del hogar. & .531 \\
\hline
\end{tabular}

Donde el factor de Satisfacción Emocional se refiere al cómo las personas expresan el amor por el otro, si se preocupa y trata de siempre estar presente, demostrándole su afecto y que disfrute estar con su pareja. Estos reactivos se refieren, por ejemplo, a la frecuencia en que me besa, la forma en que toma decisiones que nos incumben a los dos, la forma en que platica conmigo, la frecuencia con que me abraza, la sensibilidad con que responde a mis emociones, entre otros.

El factor de Satisfacción Instrumental hace énfasis en la manera práctica de llevar la relación de pareja. Esto se refiere al trato cotidiano que va desde cumplir con el compromiso sin interesarse realmente por el otro. Los reactivos en este factor fueron, por ejemplo: la atención que le pone a nuestros hijos, el trato que le da a nuestros hijos, la forma en que distribuye el dinero. Como podemos ver, estos reactivos hablan de la relación puramente de compromiso.

Posteriormente, se realizaron análisis de frecuencias por factores, para, a partir de la media, poder subdividir a los grupos por rasgos masculinos-femeninos, positivos o negativos, y áreas de satisfacción marital, siguiendo una distribución pura (aquella dada por los factores resultantes originalmente de cada una de las escalas aplicadas), o bien, factores producto de la combinación de los anteriores. Dentro de estos últimos se agregaron: Andróginos Positivos (los altos, tanto en rasgos masculinos como femeninos positivos), Andróginos Negativos (los altos, tanto en rasgos masculinos como femeninos negativos) e Indiferenciados (aquellos que, por su autodescripción, fue imposible definir con una tendencia de género). Dicho análisis se hizo por separado para hombres y mujeres, con la finalidad de conocer cuál es la distribución de los 7 factores resultantes y, con ello, responder a la primera pregunta de esta investigación. Para después, a partir de un análisis de contenido y de comparación con la literatura al respecto, poder responder a la segunda. 
La gráfica 1 permite ver que los rasgos que utilizan los hombres migrantes mexicanos, para convivir en Estados Unidos, en primer lugar son de tipo Andrógino (47.1\%), esto quiere decir que combinan sus rasgos de masculinidad y feminidad positivas, lo cual los describe como hombres autosuficientes, orientados al éxito que, al mismo tiempo, se preocupan por tener una buena relación con ellos y con los demás, mostrándose cariñosos, comprensivos y leales. En segundo plano, predominan en ellos los rasgos de Masculinidad Negativa (11.8\%) que los describen como personas que no se preocupan por ellos ni por los demás, ni se interesan por cumplir sus metas, son insociables, intolerantes, flojos, mandilones. En tercer plano, se describen con rasgos de Masculinidad Positiva (10.6\%), los cuales los refieren como personas orientadas al éxito y autosuficientes. Finalmente, en menor proporción, se encuentran tanto los hombres que se describen con rasgos de Femineidad Negativa como Positiva (ambos 8.2\%), donde los primeros son hombres que no confían en sí mismos y no pueden tomar decisiones por ellos, mostrándose miedosos, indecisos e intolerantes ante su pareja y las personas que los rodean. Los segundos, hombres que no pueden interactuar de manera adecuada, no pueden tomar decisiones, se muestran de manera insegura ante los demás y no les interesa socializar. Por último, se mostraron los hombres que no tenían ninguna tendencia por ninguno de los seis grupos, llamándolos Indiferenciados (5.9\%).

\section{GRÁFICA 1}

Distribución de rasgos de masculinidad-femineidad para hombres migrantes con pareja

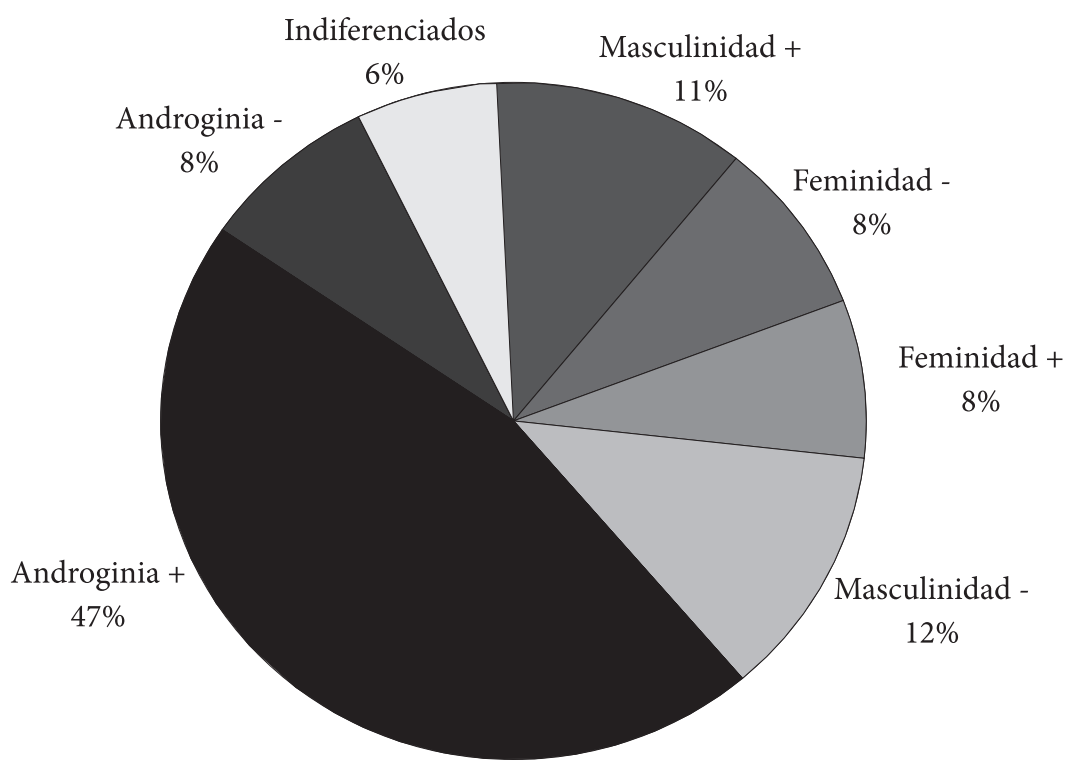

La gráfica 2 muestra los rasgos predominantes en las mujeres migrantes. En primer lugar, se observa, en la mayoría, rasgos Andróginos, esto quiere decir que combinaban sus rasgos de masculinidad y feminidad positivas. Por ello, se les puede describir como personas orientadas al éxito, con una meta, pero que son, al mismo tiempo, ca- 
riñosas, leales y comprensivas (27.9\%). En un segundo plano, se colocaron las mujeres que utilizan rasgos de Masculinidad Positiva, es decir, que se enfocan en buscar mejores oportunidades de vida, independientes, trabajadoras y respetuosas (20.9\%). En tercero, a las mujeres que no tenían ningún rasgo en particular, llamándolas Indiferenciados (16.3\%), pues presentan tanto rasgos de Femineidad Positiva (cariñosas, amables) como de Femineidad Negativa (dependientes, inestables). En cuarto lugar, paralelamente, aparecen las mujeres con rasgos femeninos, positivos y negativos por separado, pero con semejante porcentaje (11.6\%). En quinto, las mujeres que utilizaban sus rasgos de Masculinidad Negativa (9.7\%) y que se describen como: agresivas, miedosas e insociables. Por último, las mujeres que utilizaban más sus rasgos Negativos, tanto de Feminidad como de Masculinidad, las cuales son mujeres dependientes, miedosas, agresivas $\mathrm{y}$, al mismo, tiempo insociables, flojas y aprovechadas (ver gráfica 2).

\section{GRÁFICA 2}

Distribución de rasgos masculinos-femeninos en las mujeres migrantes con pareja

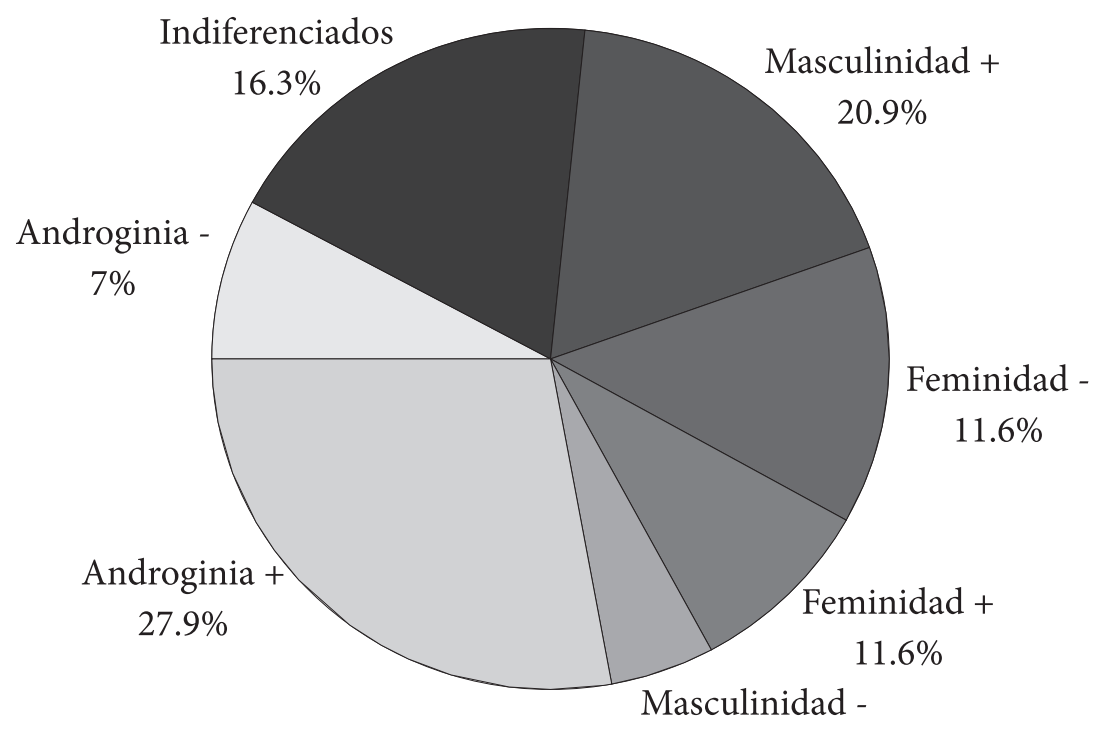

$4.7 \%$

Con respecto a la Satisfacción Marital, la gráfica 3 muestra que la mayoría de los hombres migrantes utilizan, para interactuar con su pareja, una combinación entre vínculos de tipo Emocional e Instrumental, lo que los lleva a percibirse como satisfechos en su relación marital, es decir, se reportan interesados en su relación, cariñosos, amables con los demás, preocupados por los hijos y el bienestar familiar (38.8\%). En segundo lugar, predominan los hombres que basan su vínculo matrimonial en el plano emocional, en la expresión y manejo de afectos (25.9\%). En tercer lugar, se encuentran los hombres que se relacionan, maritalmente, de una manera más instrumental que emocional, cuya preocupación va en función de mantener a la familia unida, como compromiso familiar 
y proveer lo necesario para la educación de los hijos (23.5\%). Por último, en la gráfica se visualizan los hombres que no tienen un estilo de interacción marital definido, por lo que reportan bajos niveles Instrumental y Emocional, es decir, hombres que no se involucran en la relación, ni emocional ni económicamente, simplemente dejan que el tiempo pase y no trabajan activamente en el mantenimiento y fortalecimiento de la vida en pareja y/o en familia (11.8\%).

GRÁFICA 3

Distribución por factores de vinculación matrimonial para hombres migrantes con pareja

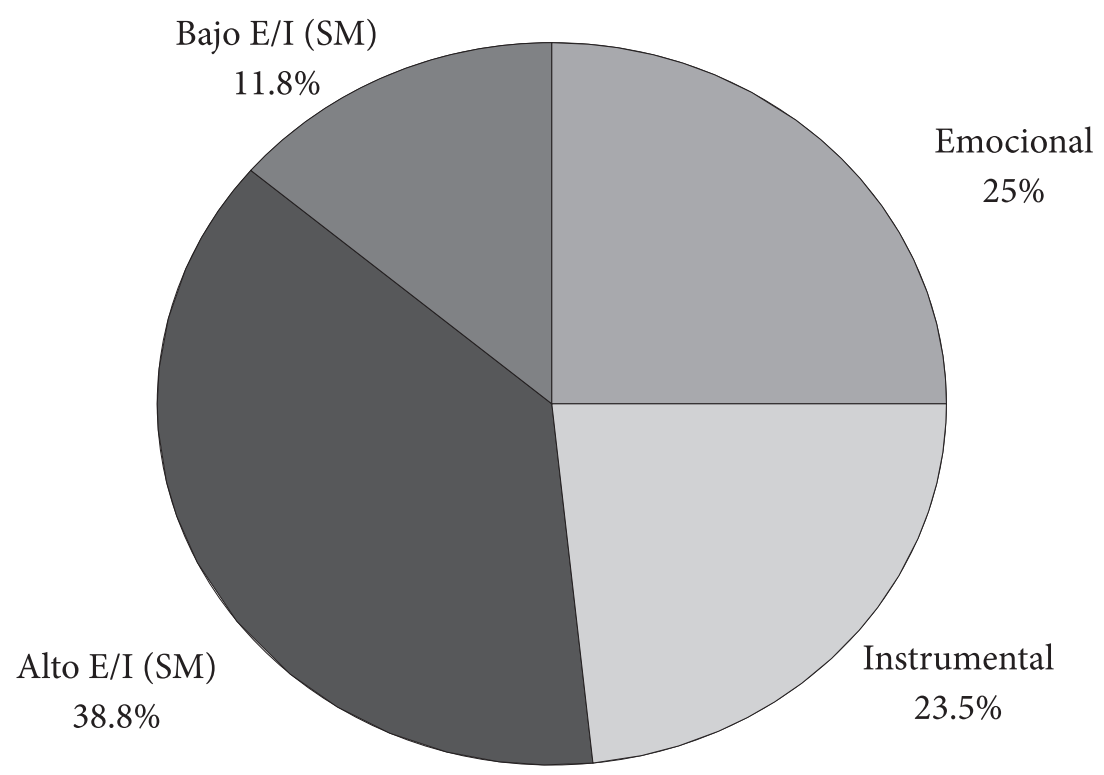

La gráfica 4 muestra los rasgos predominantes de las mujeres migrantes, en su relación marital. En primera instancia, se observan mujeres que suelen interactuar con su pareja al estilo Instrumental (39.5\%), lo cual las describe como personas interesadas en el bienestar de la familia y, por consiguiente, en todo aquello que constituya lo necesario para satisfacer las necesidades básicas de sus integrantes, más que en la demostración de afectos. En segundo lugar, se ubican las mujeres que no tiene clara la forma de vinculación con su pareja, es decir, que no se preocupan por la parte de recursos y de sostenimiento económico del hogar, ni por el bienestar socio-afectivo de la familia (34.9\%). En tercer lugar, se ubican a las mujeres contrarias a las anteriores, que consideran importante relacionarse con su pareja, tanto de manera Instrumental como Emocional, donde su preocupación se encamina a buscar el bienestar integral de la pareja y la familia, es decir, buscan que no falte nada para lograr el bienestar de sus integrantes, en un ambiente de expresión de afectos y demostración de cariño (16.3\%). Por último, se observan a las mujeres que hacen énfasis en el Vínculo Emocional en su relación de pareja, es decir, mujeres que consideran que con el amor se puede todo (9.3\%). 
GRÁFICA 4

Distribución por factores de vinculación matrimonial

para mujeres migrantes con pareja

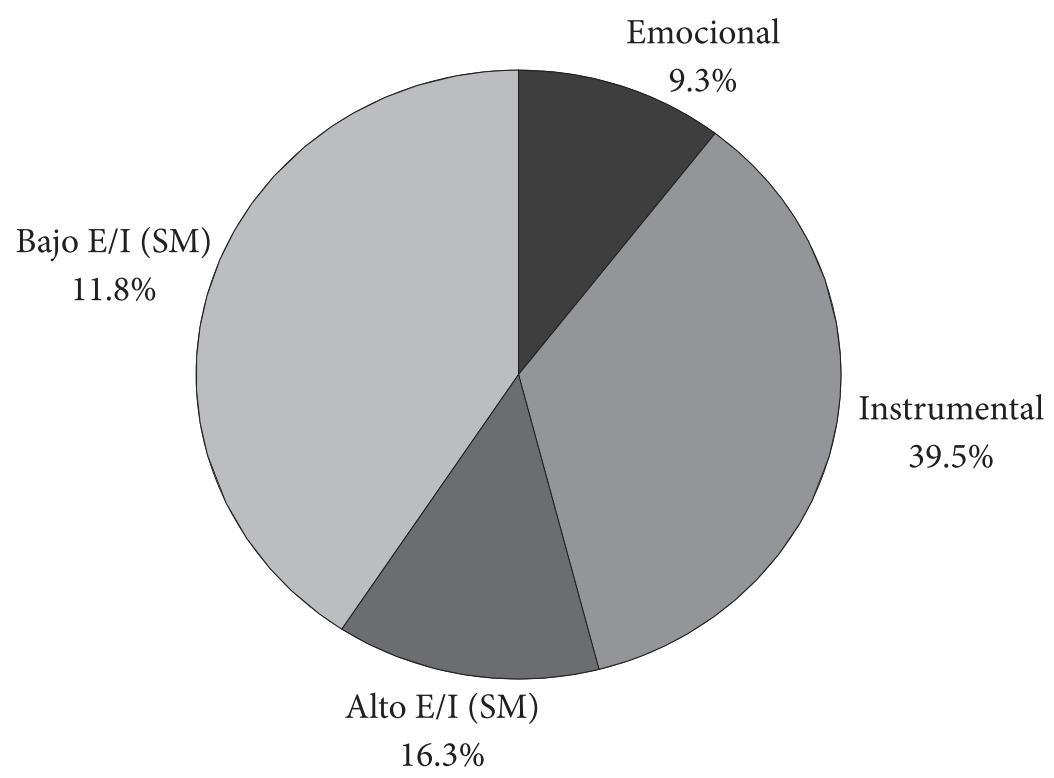

En resumen, los datos de la gráfica 1 permiten ilustrar que los rasgos de masculinidad-feminidad que predominan entre los miembros de la pareja migrante son, en el sentido «puro»: Masculinidad Positiva, Feminidad Positiva, Feminidad Negativa y Masculinidad Negativa. De la combinación de estos últimos, se observa que ni los hombres ni las mujeres continúan siendo puramente masculinos o femeninos (según se trate); por el contrario, toman lo positivo de ambos rasgos de género y, culturalmente, tanto hombres como mujeres migrantes, en primera instancia, se autodescriben como Andróginos. En segundo lugar, se puede observar que, mientras los hombres se adjudican rasgos de tipo Masculino Negativo, las mujeres toman lo Positivo de este mundo como predominante.

Ahora bien, como una forma de análisis con respecto a la influencia que el contexto cultural y, en particular, el proceso de migración tiene sobre la manera en que la pareja migrante percibe y evalúa sus relaciones interpersonales, las gráficas 3 y 4 muestran un cambio cultural en cuanto a la percepción que hombres y mujeres hacen de su relación matrimonial. Por un lado, ellos se reportan como satisfechos en ambas áreas, emocional e instrumental, seguidos por un aumento sustancial en su expresividad y manifestación de emociones con la pareja. En cambio, las mujeres, en primer lugar, reportaron un nivel significativamente alto en vinculación instrumental, e insatisfechas, maritalmente hablando.

Más adelante, se hizo un Análisis de Varianza por sexo para cada uno de los factores resultantes de las escalas aplicadas. Las tablas 4 y 5 muestran que dichos factores, que resultan estadística y significativamente diferentes para hombres y mujeres migrantes, son: la Feminidad Negativa, así como rasgos de Masculinidad-Feminidad, por un lado, y el 
factor de Satisfacción Emocional, por otro. En la primera, se puede apreciar que son las mujeres las que suelen adoptar más rasgos de Feminidad Negativa: acostumbran conducirse como indecisas, inseguras, miedosas, inestables. En la segunda, se observa como ellos son los que, dentro de la vida marital, buscan una vinculación con la pareja cargada de emociones y formas de intercambio, a través de la forma y frecuencia del contacto físico.

TABLA 4

Análisis de varianza por sexo para cada uno de los factores de la escala de Roles de Género de Polanco (2004), para una muestra de individuos migrantes mexicanos con pareja

\begin{tabular}{|c|c|c|c|c|}
\hline ROLES DE GÉNERO DE: & MASCULINIDAD + & MASCULINIDAD - & FEMINEIDAD + & FEMINEIDAD - \\
\hline Hombres & $\mathrm{x}=53.15$ & $\mathrm{X}=15.23$ & $\mathrm{x}=44.66$ & $\mathrm{x}=13.46$ \\
\hline Mujeres & $\mathrm{x}=53.60$ & $\mathrm{x}=15.11$ & $\mathrm{x}=42.83$ & $\mathrm{X}=15.43$ \\
\hline $\mathrm{F}(1 / 123)=$ & .054 & .025 & 1.278 & 4.576 \\
\hline $\mathrm{p}=$ & .817 & .874 & .260 & .035 \\
\hline
\end{tabular}

Nota: Masculinidad += Masculinidad Positiva, Masculinidad - = Masculinidad Negativa, Femineidad $+=$ Femineidad Positiva, Femineidad $-=$ Femineidad Negativa

TABLA 5

Análisis de varianza por sexo para los dos factores resultantes del Inventario Multifacético de Satisfacción Marital, para una muestra de individuos migrantes mexicanos con pareja

\begin{tabular}{|c|c|c|}
\hline VINCULACIÓN MATRIMONIAL DE: & EMOCIONALIDAD & INSTRUMENTALIDAD \\
\hline Hombres & $\mathrm{x}=33.8$ & $\mathrm{x}=9.37$ \\
\hline Mujeres & $\mathrm{x}=25.51$ & $\mathrm{x}=8.11$ \\
\hline $\mathrm{F}(1 / 123)=$ & 16.87 & 2.65 \\
\hline $\mathrm{p}=$ & .000 & .106 \\
\hline
\end{tabular}

Finalmente, para poder responder a la tercera pregunta, «¿cuál de las combinaciones entre rasgos masculinos-femeninos predominantes favorece la satisfacción marital en una muestra de migrantes mexicanas hacia los EU?», se procedió a realizar un Análisis de Regresión Lineal por el Método Inductorio por Sexo, para los 4 factores resultantes de la escala de Roles de Género, tomando, como variable dependiente, la Satisfacción Marital en sus dos características: Satisfacción Emocional e Instrumentalidad. Cabe aclarar que, aunque se siguió este mismo procedimiento con los tres factores resultantes de la combinación entre los primeros - Andróginos Positivos, Andróginos Negativos e Indiferenciados-, los resultados no fueron estadísticamente significativos, por lo que se reportan más adelante. Previo a esos análisis de regresión, se realizaron Correlaciones Producto-Momento de Pearson por sexo y factores.

La tabla 6 muestra, en el caso de los hombres, una correlación positiva y significativa entre feminidad positiva y, por separado, la negativa, ambas con la vinculación matrimonial de emoción. Para las mujeres, las correlaciones que resultaron estadísticamente 
significativas se dieron entre masculinidad negativa y feminidad positiva, por separado con la vinculación matrimonial de emoción, la feminidad positiva y la vinculación matrimonial instrumental.

TABLA 6

Correlaciones Producto-Momento de Pearson para Hombres y Mujeres entre rasgos Masculinos y Femeninos y Estilos de Vinculación Emocional e Instrumental en una muestra de individuos mexicanos migrantes con pareja

\begin{tabular}{|c|c|c|c|c|}
\hline \multirow{3}{*}{$\begin{array}{l}\text { RASGOS MASCULINOS Y } \\
\text { FEMENINOS }\end{array}$} & \multicolumn{4}{|c|}{ ESTILOS DE VINCULACIÓN MATRIMONIAL } \\
\hline & \multicolumn{2}{|c|}{ INSTRUMENTAL } & \multicolumn{2}{|c|}{ EMOCIONAL } \\
\hline & HOMBRES & MUJERES & HOMBRES & MUJERES \\
\hline Masculinidad - & $\begin{array}{c}\mathrm{x}=.059 \\
(.59)\end{array}$ & $\begin{array}{l}\mathrm{X}=.255 \\
(.10)\end{array}$ & $\begin{array}{l}\mathrm{X}=.152 \\
(.16)\end{array}$ & $\begin{aligned} \mathrm{X} & =.399^{* *} \\
& (.00)\end{aligned}$ \\
\hline Femineidad + & $\begin{array}{c}\mathrm{X}=.134 \\
(.22)\end{array}$ & $\begin{array}{c}\mathrm{X}=.512^{* *} \\
(.001)\end{array}$ & $\begin{array}{c}\mathrm{X}=.247^{* *} \\
(.02)\end{array}$ & $\mathrm{x}=.478^{* *}$ \\
\hline Femineidad - & $\begin{array}{l}\mathrm{X}=.082 \\
(.46)\end{array}$ & $\begin{array}{c}\mathrm{X}=-.058 \\
(.73)\end{array}$ & $\begin{array}{c}\mathrm{X}=.224^{* *} \\
(.04)\end{array}$ & $\begin{array}{c}\mathrm{x}=-.102 \\
(.54)\end{array}$ \\
\hline Masculinidad + & $\begin{array}{l}\mathrm{X}=.027 \\
(.25)\end{array}$ & $\begin{array}{l}\mathrm{X}=.284 \\
(.07)\end{array}$ & $\begin{array}{c}\mathrm{X}=.340^{* *} \\
(.00)\end{array}$ & $\begin{array}{c}\mathrm{x}=.063 \\
(.69)\end{array}$ \\
\hline
\end{tabular}

Nota: Masculinidad $-=$ Masculinidad Negativa, Femineidad $+=$ Femineidad Positiva, Femineidad $-=$ Femineidad Negativa y Masculinidad $+=$ Masculinidad Positiva. ${ }^{*} \mathrm{p}=.05,{ }^{* *} \mathrm{p}=.001$

TABLA 7

Análisis de Regresión Lineal por Método Introductorio por sexo tomando, como variable dependiente, los factores que integran la escala de Satisfacción Marital: Emocional e Instrumental en una muestra de individuos mexicanos migrantes con pareja

\begin{tabular}{|c|c|c|c|}
\hline \multirow{2}{*}{} & \multicolumn{2}{|c|}{ MUJERES } & HOMBRES \\
\cline { 2 - 4 } & \multicolumn{2}{|c|}{ VARIABLE DEPENDIENTE: VINCULACIÓN MATRIMONIAL } \\
\hline MODELO PREDICTIVO & EMOCIONAL & INSTRUMENTAL & EMOCIONAL \\
\hline Masculinidad + & -.133 & .051 & .356 \\
\hline Femineidad - & -.130 & -.020 & -.031 \\
\hline Femineidad + & .506 & .114 & .151 \\
\hline Masculinidad - & 1.363 & .332 & $(4 / 80) 3.53^{*}$ \\
\hline F= & $(4 / 36) 3.670^{* *}$ & .5069 & .395 \\
\hline R= & .5619 & .256 & .156 \\
\hline R2= & .314 & 4.485 & 8.770 \\
\hline B= & -8.337 & & \\
\hline
\end{tabular}

Nota: Masculinidad $-=$ Masculinidad Negativa, Femineidad $+=$ Femineidad Positiva, Femineidad $-=$ Femineidad Negativa y Masculinidad $+=$ Masculinidad Positiva.

$$
{ }^{*} \mathrm{p}=.011,{ }^{* *} \mathrm{p}=.01,{ }^{* * *} \mathrm{p}=.04
$$


La tabla 7 permite visualizar que es la masculinidad positiva la que tiende a predecir, tanto para hombres como para mujeres, con mayor fuerza el tipo de vinculación matrimonial, pero sólo a nivel de expresión de emociones, afectos y sentimientos hacia el cónyuge.

\section{DISCUSIÓN}

De acuerdo con los resultados obtenidos a través de las respuestas de la Escala de Roles de Género de Polanco (2004: 122-132), así como las del Inventario Multifacético de Satisfacción Marital de Cortés (1994: 118-119), aplicados a individuos migrantes mexicanos con pareja, se pudo observar que los rasgos de Masculinidad-Feminidad predominantes entre estas parejas son cuatro: Masculinidad Positiva, Feminidad Positiva, Feminidad Negativa y Masculinidad Positiva, tal como se muestra en la literatura (Brehm, 1992: 136-137; Carrillo, Cortés, Flores y Reyes, 2000: 118-119; Díaz-Loving y Rocha, 2004: 87; Hoffman, 2000: 476; Polanco, 2004: 134-137).

Un análisis de las combinaciones de dichos rasgos permiten ver que ni los hombres ni las mujeres siguen un rasgo puramente definido de género, puesto que muchos ya han adoptado lo positivo de ambos rasgos y, en términos culturales, tanto mujeres como hombres migrantes se definen como Andróginos Positivos.

En segundo lugar, el rasgo que predominó fue el de Masculinidad Negativa, en hombres, y Masculinidad Positiva, en mujeres, que es contrario a lo esperado, de acuerdo con la literatura clásica sobre género. Esto es así, como afirma Díaz-Loving, et al. (1994: 138-139), porque, con el paso del tiempo y la evolución cultural, los rasgos masculinos y femeninos, a pesar de estar claramente definidos para hombres y mujeres, han hecho que las mujeres desarrollen más su masculinidad, como consecuencia de que sus maridos migren al otro lado de la frontera y ellas tengan que cumplir con las funciones de madre y padre a la vez. Por el lado de los hombres, como resultado del estado de soledad a la que el proceso de migración los conduce, ellos han tenido que aprender a expresar sus emociones y trabajar arduamente, para generar un cambio en su estilo de vida e ir en busca de mejores oportunidades (Sánchez de la Barquera, 1997: 5).

Hablando de Satisfacción Marital, los resultados obtenidos muestran que estas parejas viven un cambio cultural en la percepción que tienen de su relación matrimonial. Los hombres que reportan estar satisfechos en la vinculación emocional y en la instrumental, experimentan un aumento sustancial en su expresividad y manifestación de emociones para con la pareja. Mientras que las mujeres reportan, en primer lugar, una mayor vinculación instrumental y, en segundo, se describen insatisfechas, maritalmente hablando, por el hecho de tener que trabajar y cumplir con un doble o triple rol (ser trabajadoras, esposas y/o madres). Esta situación les genera altos índices de estrés, lo que repercute en su dinámica y ajuste marital (Campbell \& Snow, 1992: 86-87; Fischer \& Good, 1997: 167). Esto confirma, asimismo, lo que Brehm (1992: 136-137) y Hoffman (2000: 476) refieren sobre el hecho de que el poseer rasgos positivos masculinos favorece la Satisfacción Marital en los hombres, más no en las mujeres. Podemos decir que en este estudio sobre parejas, con uno de sus miembros migrante, hemos encontrado que la masculinidad positiva «pura» está, principalmente, reportada en las mujeres. 


\section{BIBLIOGRAFÍA}

BrenM, S. L. (1992), «The measurement of psychological androgyny», Journal of Consulting and Clinical Psychology, 42, pp. 133-145, 155-162, 404-415.

Bustos Romero, Olga (1994), «La perspectiva de género y la división sexual del trabajo», La Psicología Social en México, Vol. v, Amepso, México, pp. 755-761.

CAMPBell, J. L. \& Snow, B. M. (1992), «Gender role conflict and family environment as predictors of men's marital satisfaction», Journal of Family Psychology, 6, pp. 84-87.

Carrillo, C., Cortés, L., Flores, M., \& Reyes, I. (2000), «Niveles de Expresividad-Instrumentalidad en Hombres y Mujeres», La Psicología Social en México, Vol. VIII, Amepso, México, pp. 114-120.

Cortés Martínez, S., Reyes Domínguez, D., Díaz-Loving, R., Rivera Aragón, S. y Monjaraz Carrasco, J. (1994), «Elaboración y Análisis Psicométrico del Inventario Multifacético de Satisfacción Marital (IMSM)», La Psicología Social en México, Vol. v, Amepso, México, pp. 123-130.

Cournoyer, R. J. \& Mahalik, J. R. (1995), «Cross-sectional study of gender role conflict examining college-aged and middle-aged men», Journal of Counseling Psycho$\log y, 42$, pp. 11-19.

Díaz-Guerrero, R. (1994), Psicología del Mexicano. Descubrimiento de la Etnopsicología, Trillas, México, pp. 135-181.

DíAz-Loving, R. (1996), «Rasgos de masculinidad-feminidad. Efectos sobre la satisfacción familiar en hombres y mujeres», La Psicología Social en México, Vol. VI, pp. 268-274.

DíAz-Loving, R., Rivera Aragón, S. y Sánchez Aragón, R. (1994), «Género y Pareja», Revista de Psicología Contemporánea, Vol. 1 (2), pp. 4-15.

DíAz-Loving, R., Rivera Aragón, S. y Sánchez, Aragón, R. (2001), «Rasgos Instrumentales (masculinos) expresivos (femeninos) normativos (típicos e ideales) en México», Revista Latinoamericana de Psicología, Vol. 33, pp. 131-139.

DíAz-Loving, R. y Rocha Sánchez, T. (2004), «Replica y Extensión de la Escala de Rasgos de Instrumentalidad y Expresividad», La Psicología Social en México, Vol. x, pp. 80-88, Amepso, México.

DíAz-Loving, R., Ruiz, B. P., Cárdenas, R. M., Alvarado, H. V. y Reyes, R. D. (1994), «Masculinidad-Femineidad y Satisfacción Marital: Correlaciones e implicaciones», La Psicología Social en México, Vol. v, AMEPso, México, pp. 138-145.

Diener, E, \& Sun, E. (1998), «The shifting basis of life satisfaction judgments across cultures: Emotions versus norms», Journal of Personality and Social Psychology, Vol. 74 (2), pp. 482-493.

Finch, B. K., Catalano, R. C., Novaco, R. W. \& Vega, W. A. (2003), «Employment Frustration and Alcohol Abuse/Dependence Among Labor Migrants in California», Journal of Immigrant and Minority Health, Vol. 5 (4), pp. 181-186.

Fischer, A. R. \& Good, G. E. (1997), «Men and Psychotherapy: An investigation of alexithymia, intimacy and masculine gender roles», Psychotherapy, 36, pp. 160-170. 
Frone, M. R., Yardley, J. K. \& Markel, K. S. (1997), «Developing and testing an integrative model of the work-family interface», Journal of Vocational Behavior, pp. 145-167.

Gilligan, C. (1982), In a different voice, Harvard University Press, Cambridge, MA, pp. $152-173$

Hayes, J. A. \& Mahalik, J. R. (2000), «Gender role conflict and psychological distress in male counseling center clients», Psychology of Men \& Masculinity, 1, pp. 116-125.

Hoffman, Rose M., Borders, L. D. \& Hattie, J. A. (2000), «Reconceptualizing Femininity and Masculinity: From Gender Roles to Gender Self-Confidence», Journal of Social Behavior \& Personality, Vol. 15, Fascículo 4.

Kanaiaupuni, S. M. (2000), «Reframing the Migration Question: An Analysis of Men, Women, and Gender in Mexico», Social Forces, Vol. 78, Issue 4, pp. 1311-1347.

Maldonado Rubí, M. C. y García y García, B. E. (1994), «La educación de las mujeres en México», La Psicología Social en México, Vol. v, México, AMePso, pp. 749-754.

Parrado, Emilio A. \& Flippen, C. A. (2005), «Migration and Gender among Mexican Women», American Sociological Review, Vol. 70, (4), pp. 606-632.

Polanco, G. (2004), Los roles sociales en la cultura, tesis doctoral no publicada, Facultad de Psicología, Universidad Nacional Autónoma de México, México D.F.

Rocha, S. T. (2004), Roles de Género en los adolescentes mexicanos y rasgos de masculinidad-feminidad, tesis de licenciatura, Facultad de Psicología, Universidad Nacional Autónoma de México, México D.F.

Sánchez de la Barquera Vera, L. B. (1997), La migración laboral e indocumentada mexicana a los Estados Unidos, tesis de licenciatura, Universidad Iberoamericana, México.

Sharpe, M. J., Heppner, P. P. \& Dixon, W. A. (1995), «Gender role conflict, instrumentality, expressiveness, and well-being in adult men», Sex Roles, Vol. 33 (1), pp. 1-18.

Veenhoven, R. (2005), «Is life getting better?: How long and happy do people live in modern society?», European Psychologist, 10, pp. 330-343. 